\title{
The Morphology of Anomalous Cosmic Rays in the Outer Heliosphere
}

\author{
C.D. Steenberg \\ California Institute of Technology, Mail code 220-47, Pasadena, CA, 91125, USA
}

\begin{abstract}
The well established cosmic ray transport equation describes the physics of cosmic ray modulation in the heliosphere and is thought to be complete. Its solution for anomalous cosmic rays has special characteristics due to the local acceleration of these particles at the solar wind termination shock. Some of these characteristics are demonstrated here, namely effects by the strength of the shock, shock drift, the cutoff in the spectrum at the shock, species scaling, drift, and ionization.
\end{abstract}

\section{INTRODUCTION}

In a test particle approach the distribution of cosmic ray particles in the heliosphere can be described by Parker's transport equation (TPE) [18]. In a recent overview of the state of modulation theory [5] it is argued that the physics contained in this equation is sufficiently complete to describe cosmic ray modulation processes. The problem that remains is to develop a detailed description of the time-dependent characteristics of the solar wind plasma causing the modulation of cosmic rays. This description needs to be put into the TPE to produce a realistic modulation model. It is expected that such a realistic model would consist of a system of equations, including an MHD description of the solar wind plasma.

The characteristics of the modulation of cosmic rays is determined to a large extent by the "source" or boundary spectrum that is modulated. The source of anomalous cosmic rays (ACRs) is widely believed to be the the acceleration of so-called pick-up ions [6] at the solar wind termination shock (SWTS) as proposed by [16]. In this paper solutions of Parker's equation will be used to demonstrate the unique features of ACRs and their dependence on the location, strength, diffusion parameters at the SWTS, ionization, and drift effects.

A set of modulation parameters with simple spatial and energy dependence is chosen to demonstrate these features as clearly as possible. More realistic parameters would yield results that are quantitatively different, but still qualitatively similar. This paper is an extension of the work published in $[17,20]$.

\section{TRANSPORT AND ACCELERATION OF COSMIC RAYS}

The transport equation contains the modulation effects of convection, diffusion, drifts in the HMF, adiabatic energy losses, and acceleration at the SWTS. In terms of the omnidirectional distribution function $f$, the TPE can be written as

$$
\frac{\partial f}{\partial t}=\nabla \cdot(\mathbf{K} \cdot \nabla f-\mathbf{V} f)+\frac{1}{3}(\nabla \cdot \mathbf{V}) \frac{\partial f}{\partial \ln P}+Q
$$

where $P=p c / Z$ is particle rigidity in terms of momentum, $p$, the speed of light, $c$, and ionic charge $Z$. Diffusion and drift effects are described by the diffusion tensor, $\mathbf{K}, \mathbf{V}$ is the solar wind vector, and $Q$ represents sources/sinks of particles. The diffusion tensor contains anti-symmetrical terms quantifying drift $\left(\kappa_{T}\right)$ and diffusion parallel and perpendicular to the mean magnetic field $\left(\kappa_{\|}, \kappa_{\perp 1}, \kappa_{\perp 2}\right)$.

The diffusive shock (first order Fermi) acceleration mechanism produces spectra at the SWTS (e.g. [3]) of the form

$$
f\left(r_{s}, P\right)=q P^{-q} \int_{0}^{P} \frac{Q\left(P^{\prime}\right)}{V_{r}} P^{\prime q-1} d P^{\prime}
$$

In the special case of a mono-energetic source spectrum $Q(P, r)=Q_{0} \delta\left(P-P_{i}\right) \delta\left(r-r_{s}\right)$, where $\delta$ is the Dirac-delta function, injected at rigidity $P_{i}$, into a steady, plane SWTS with compression ratio $s$, positioned at a distance $r_{s}$ from the sun, this reduces to

$$
f\left(r_{s}, P\right) \propto P^{-q}
$$


with spectral index $q=3 s /(s-1)$. In terms of intensity, $j_{T} \propto P^{2} f$, and kinetic energy, $T$, this accelerated spectrum reduces to

$$
j_{T} \propto T^{-(q-2) / 2} \text { or } j_{T} \propto T^{-(q-2)}
$$

in the non-relativistic and relativistic limits respectively.

This power-law accelerated spectrum will extend to a maximum energy limited by the available acceleration time, the finite dimensions of the shock, adiabatic energy losses (e.g. [3, 9]), and also ionization [11]. Considering the short acceleration time needed to extend the power law to ACR energies [10], compared to the long-lived nature of the SWTS, and the length of a solar cycle, the acceleration time is not thought to be responsible for limiting the maximum energy gain by ACRs at the quasiperpendicular SWTS.

Instead, particles are expected [3] to escape from a spherical SWTS without further acceleration when the diffusive length scale becomes comparable to the radius of curvature of the shock

$$
\frac{\kappa_{r r}}{V} \sim r_{s}
$$

Adiabatic deceleration will decrease this maximum energy, as it competes with the diffusive shock acceleration mechanism.

The maximum energy a particle can attain by acceleration at the SWTS can also be estimated in an interesting special case as follows: by noting [8] that in absence of diffusion, for a purely perpendicular shock, and for particles that can not escape from the shock, the energy gain due to first order Fermi acceleration is equivalent to an energy gain of a charged particle in a potential field $\mathbf{E}=-\mathbf{V} \times \mathbf{B}$. In this case a positively charged particle gains energy as it drifts along the shock from the equator to the pole (pole to equator) in the positive (negative) drift cycle. The maximum energy gain is then limited by the potential difference between equator and pole. This upper limit was estimated to be a total energy of $\sim 300$ $\mathrm{MeV}$ by [11] and references therein.

\section{THE POWER LAW SPECTRUM AND CUTOFF}

It is instructive to to solve (1) for a spherically symmetric heliosphere to illustrate the power law and its cutoff at the SWTS.

Using the numerical model of [22], a baseline solution of (1) is obtained for 140 radial intervals between a re- flecting inner boundary at $r_{i}=0.05 \mathrm{AU}$ and outer free escape boundary $r_{b}=120 \mathrm{AU}$, with a discontinuous, strong shock at $r_{s}=90 \mathrm{AU}$ [23]. A diffusion mean free path perpendicular to the SWTS of $\lambda_{r r}=3 \kappa_{r r} / v=\lambda_{r r 0} P / P_{0} V / V_{0}$ AU is used, with $P_{0}=1 \mathrm{GV}$, and $v$ the particle speed in the rest frame of the sun. The solar wind speed is $V=V_{0}$, with $V_{0}=400 \mathrm{~km} / \mathrm{s}$ inside the shock, and $V=V_{0}\left(r_{s} / r\right)^{2} / \mathrm{s}$ outside the shock. A mono-energetic spectrum of particles are injected at a rigidity $P$, with the rigidity domain divided into 400 logarithmically spaced intervals. The model is run for a total of 4.8 years, after which the solution has reached a stationary state.

For a baseline solution $\mathrm{ACR} \mathrm{O}^{+}$, with atomic mass to charge ratio $A / Z=16$, is modulated by setting $\lambda_{r r 0}=$ 0.5 , with $P_{i}=0.2 \mathrm{GV}(72 \mathrm{keV} / \mathrm{nuc})$, and accelerated by a strong $(s=4)$ shock. The resulting spectrum at the SWTS is shown by the solid line in Figure 1 (top). This spectrum has the expected form of a power law $T^{-1}$ at low energies, with a cutoff at $5.3 \mathrm{MeV} / \mathrm{nuc}$, using a $1 / e$ deviation from the power law as a cutoff criterion.

Also shown in Figure 1 are two parameter variations relative to the baseline: (i) Changing only the compression ratio of the shock to $s=2.5$, results in a shock spectrum with a power law of $T^{-1.5}$ (dashed line), as expected from (4). Note that the cutoff still occurs at $5.3 \mathrm{MeV} /$ nuc. (ii) Increasing the mean free path to $\lambda_{r r 0}=1.0$ results in a shock spectrum (dash-dotted line) identical to the the baseline, except that the cutoff energy is reduced by a factor of two, to $2.65 \mathrm{MeV} / \mathrm{nuc}$.

In the bottom panel of Figure 1 the modulated spectra at $60 \mathrm{AU}$ show that the spectral peak for case (i) is moved to slightly lower energies, due to stronger modulation which stems from the steeper spectrum at the shock. In case (ii) the spectral peak is shifted to lower energies by a factor of approximately two, with a lower level of modulation than in the baseline solution.

It was found that for a wide range of parameters [17, $20]$ that the cutoff in the shock spectrum occurs where

$$
\frac{V r_{s}}{\kappa_{r r}}=C_{c}
$$

where $C_{c} \approx 4.5$ for the above solutions, using the $1 / e$ criterion to determine the cutoff energy. This number is higher than the lower limit of $C_{c}=1$, in (5), which may be due to the effects of adiabatic energy losses not being taken into account in that estimate, or may simply be dependent on the criterion used to determine the cutoff energy. In comparison $[17,20]$ obtained a value of $C \approx 10$, by essentially taking the smallest detectable deviation from a power law to indicate a cutoff. 


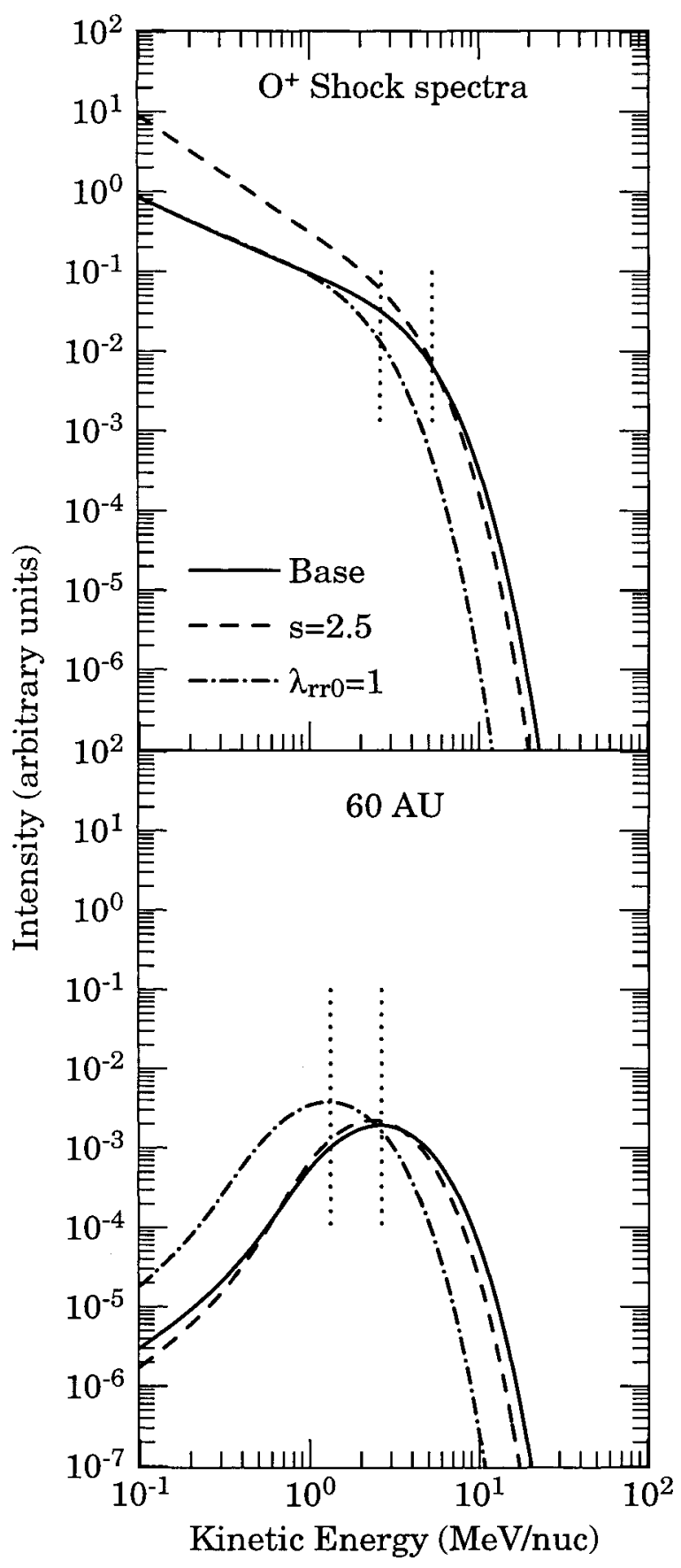

FIGURE 1. ACR $\mathrm{O}^{+}$shock spectra (top) and at $60 \mathrm{AU}$ (bottom) for (i) baseline solution with $s=4$, (ii) $s=2.5$, (iii) solution with $\lambda_{r r 0}=1$. Vertical lines (dots) indicate the cutoff energies of the shock spectra (top), and the peak energies of the modulated spectra (bottom). Both the cutoff energy, and peak energy of the modulated spectrum in the $\lambda_{r r 0}=1$ case are lower by a factor of two. The normalization was chosen arbitrarily to emphasize the effects of the parameter variations.
More important than its value is the fact that $C_{c}$ is a constant, and that the cutoff energy can easily be determined from (6), as long as the same criterion for the cutoff is applied consistently.

\section{SPECIES SCALING}

Modulated ACR spectra for different species (elements) have very similar shapes, but have different abundances and appear to be shifted in energy per nucleon ([23] and references therein). The difference in abundances are controlled by the seed particle abundance injected into the shock, while the so-called species scaling in energy is caused by the species dependence of diffusion coefficients.

The species scaling of modulation can be derived $[4,21]$ as follows: If the effective radial diffusion coefficient for more than one species of speed $\beta c$ and rigidity $P$ has an energy dependence of $\beta P^{\gamma}$, the (non-relativistic) energy per nucleon at which spectral features occur, scale as $T_{2} / T_{1} \approx \alpha_{1} / \alpha_{2}$ in the relativistic limit, with $\alpha=A / Z$. In the non-relativistic limit, which is applicable to ACRs, the species scaling factor is

$$
T_{2} / T_{1} \approx\left(\frac{\alpha_{1}}{\alpha_{2}}\right)^{\frac{2 \gamma}{\gamma+1}}
$$

which only depends on the power $\gamma$. In this derivation it is assumed that the shock spectrum (or local interstellar spectrum in the case of GCRs) that is modulated, has the same form for both species, but scaled in energy according to the species scaling factor above. This is the case for ACR spectra accelerated at the SWTS with a power law up to $s$ characteristic cutoff energy. This cutoff is controlled by the magnitude of the radial diffusion coefficient in (6), so that the cutoff energies will scale with species in the same way as modulation.

Figure 2 demonstrates the species scaling between $\mathrm{ACR} \mathrm{O}^{+}, \mathrm{He}^{+}$, and $\mathrm{H}^{+}$by solving the TPE with the baseline parameters for these species. In the top panel vertical lines show the cutoffs in the three shock spectra at 5.3, 21.2, and 84.8 MeV/nuc, as expected from (7) for the $\gamma=1$ used in all three cases. Similar lines are also drawn in the bottom panels to show that modulated peak energies at about 2.6, 5.3, and $21 \mathrm{MeV} /$ nuc for each species, respectively.

Because the species scaling factor is a unique function of the rigidity dependence $\gamma$ in the non-relativistic limit, the observed species scaling factor may be used to determine this parameter. For Voyager 1 and 2 ACR O, 


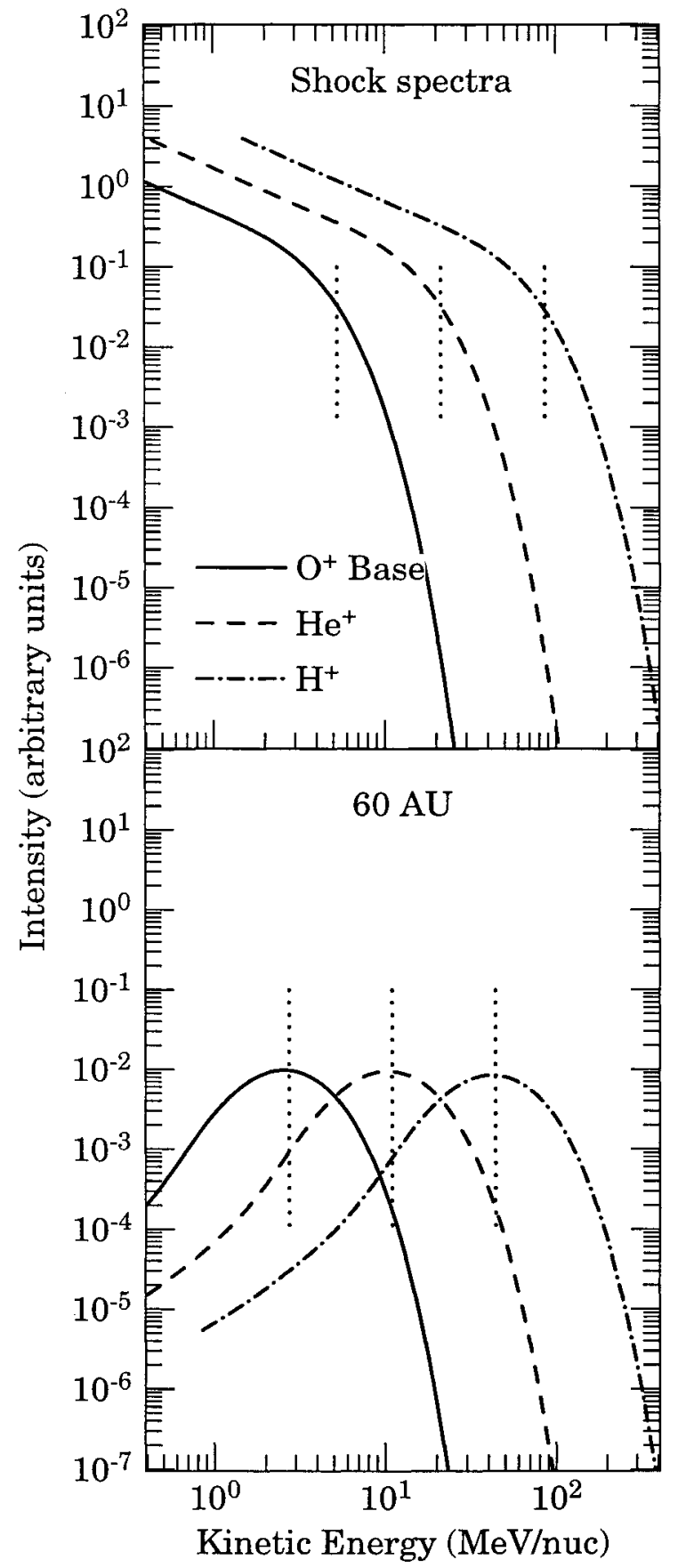

FIGURE 2. $\mathrm{ACR} \mathrm{O}^{+}$shock spectra (top) and at $60 \mathrm{AU}$ (bottom) for (i) baseline $\mathrm{O}^{+}$solution, (ii) $\mathrm{He}^{+}$, (iii) $\mathrm{H}^{+}$. Vertical lines (dots) indicate the cutoff energies of the shock spectra (top), and the peak energies of the modulated spectra (bottom). Both the cutoff energy, and peak energies for $\mathrm{He}^{+}$and $\mathrm{H}^{+}$are higher by factors of 4 and 16 respectively. The normalization was chosen arbitrarily so that the modulated intensities at $60 \mathrm{AU}$ are equal at the spectral peaks.
$\mathrm{He}$, and $\mathrm{H},[21]$ found that the species scaling factor at the spectral peaks was consistent with a value of $\gamma \approx 2$. Conversely, observed 1998 modulated spectra of several different species was fitted [19] by solutions of the TPE by using a value of $\gamma=1.6$ for rigidities up to $0.4 \mathrm{GV}$.

\section{IONIZATION OF ACRS}

Although ACRs are predominantly singly charged, recent observations $[15,14]$ show that a significant fraction may also be multiply charged. Ionization effects were consequently incorporated in a axisymmetrical timedependent modulation model [11] and subsequently applied to SAMPEX observations [2]. The method used in that model was also applied to the Steenkamp [22] model, and is summarized below.

ACRs are ionized by collisions with heliospheric neutral hydrogen [11]. If the ionization rates of ACRs are known, the source/sink function $Q_{i}^{q}$ in (1) for each charge state, $q$, is given by

$$
Q_{i}^{q}(\mathbf{r}, P, t)=n_{H}\left[R^{q-1}(T) f^{q-1}-R^{q}(T) f^{q}\right]
$$

where $R^{q}(T)$ is the ionization rate for charge state $q$, and $n_{H}$ is the neutral hydrogen density. If the speed of the neutral hydrogen atoms is negligible relative to the speed of the ACR ions, and the particle distribution $f^{q}$ is isotropic, the ionization rate is given by the product of the collision cross section and the ion speed,

$$
R^{q-1}(T)=\sigma^{q}(T) v=\sigma^{q}(T) \beta c
$$

with $\sigma^{q}$ the ion-hydrogen ionization cross section.

To model the modulation of ACRs of $N$ charge states, (1) must be solved for a system of $N$ equations with the correct ionization source/sink terms:

$$
\begin{aligned}
\frac{\partial f^{1}}{\partial t^{2}} & =\ldots-Q_{i}^{1}(\mathbf{r}, P, t) \\
\frac{\partial f^{2}}{\partial t} & =\ldots-Q_{i}^{2}(\mathbf{r}, P, t) \\
& \vdots \\
\frac{\partial f^{N}}{\partial t} & =\ldots-Q_{i}^{N}(\mathbf{r}, P, t)
\end{aligned}
$$

Each charge state undergoes modulation due to convection, diffusion, drifts, adiabatic energy losses, acceleration at the shock, with an additional sink term to account for ionization losses. For the fully ionized state, $Q_{i}^{N}(\mathbf{r}, P, t)=0$.

Assuming that only singly charged $\mathrm{O}^{+}$is injected at the shock, we repeat the baseline solution for ACR O 
and calculate the spectra and relative abundances of all 8 charge states, with the results shown in Figures 4 and 5. A neutral hydrogen density of $n_{H}=0.115$, an estimate for the value at the SWTS [7], was used throughout the heliosphere. Ionization cross sections, $\sigma^{q}$, from [1] for $O$, shown in Figure 5, have maxima in the energy range of $\sim 100$ to $\sim 200 \mathrm{keV} /$ nuc for charge states 1 to 5 , and $\sim 1$ $\mathrm{MeV} / \mathrm{nuc}$ for charge states 6 and 7 . An injection energy of $180 \mathrm{keV} /$ nuc is used, instead of $72 \mathrm{keV} /$ nuc in the baseline solution, for stability reasons, and is sufficiently low to allow a realistic demonstration.

Firstly, note that the $\mathrm{O}^{+}$spectra are only slightly depleted relative to the no-ionization baseline solution, with the depletion increasing with energy. At low energies, the $T^{-1}$ power law is also still clearly present at the shock. This indicates that the source of particles due to acceleration is large relative to the sink due to ionization in (8). Secondly, there is also a clear trend that the abundance of a particular charge state $\mathrm{O}^{N+}$ relative to $\mathrm{O}^{(N-1)+}$ decreases with increasing charge, $N$ : At $60 \mathrm{AU}, \mathrm{O}^{2+}$ makes up $5 \%$ to $10 \%$ of the total in the energy range between 1 and $10 \mathrm{MeV} /$ nuc, where the peak intensity occurs. In this energy range, $\mathrm{O}^{3+}$ contributes $0.1 \%$ to $0.5 \%$ of the total.

At the shock, the spectra of multiply charged ACRs do not have the $T^{-1}$ power law at low energies. This

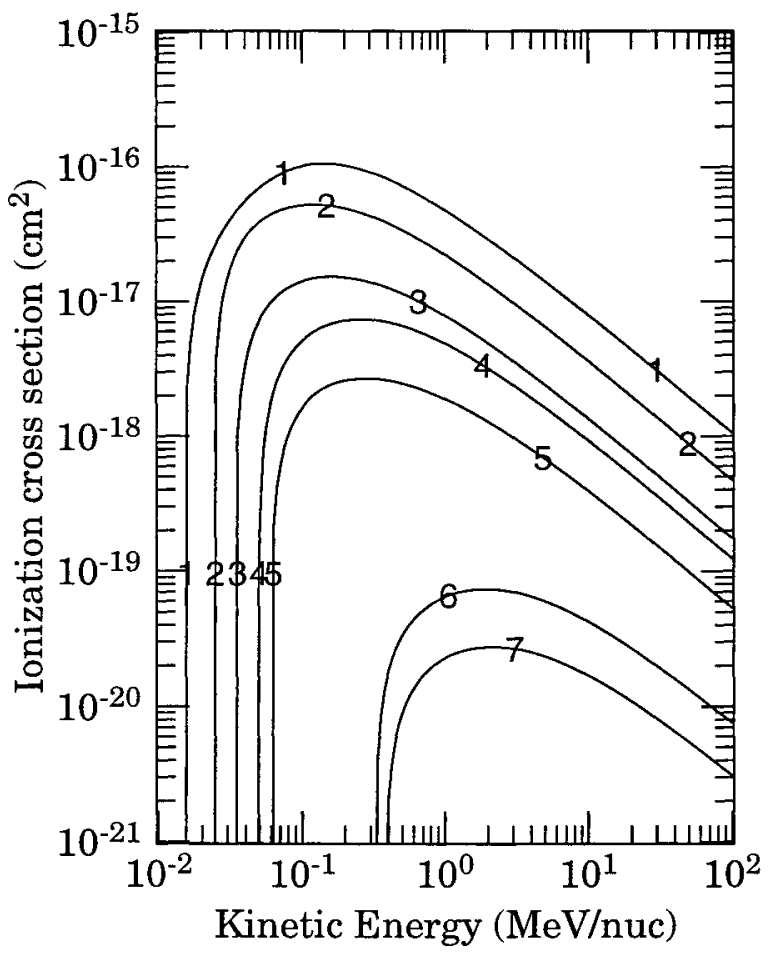

FIGURE 3. Ionization cross sections for charge states 1 to 7 of oxygen, from [1]. indicates that the majority of these particles are the products of ionization of lower charge states, rather than being ionized mainly at low energies and then accelerated. As expected from (6), the multiply charged ACRs are accel-

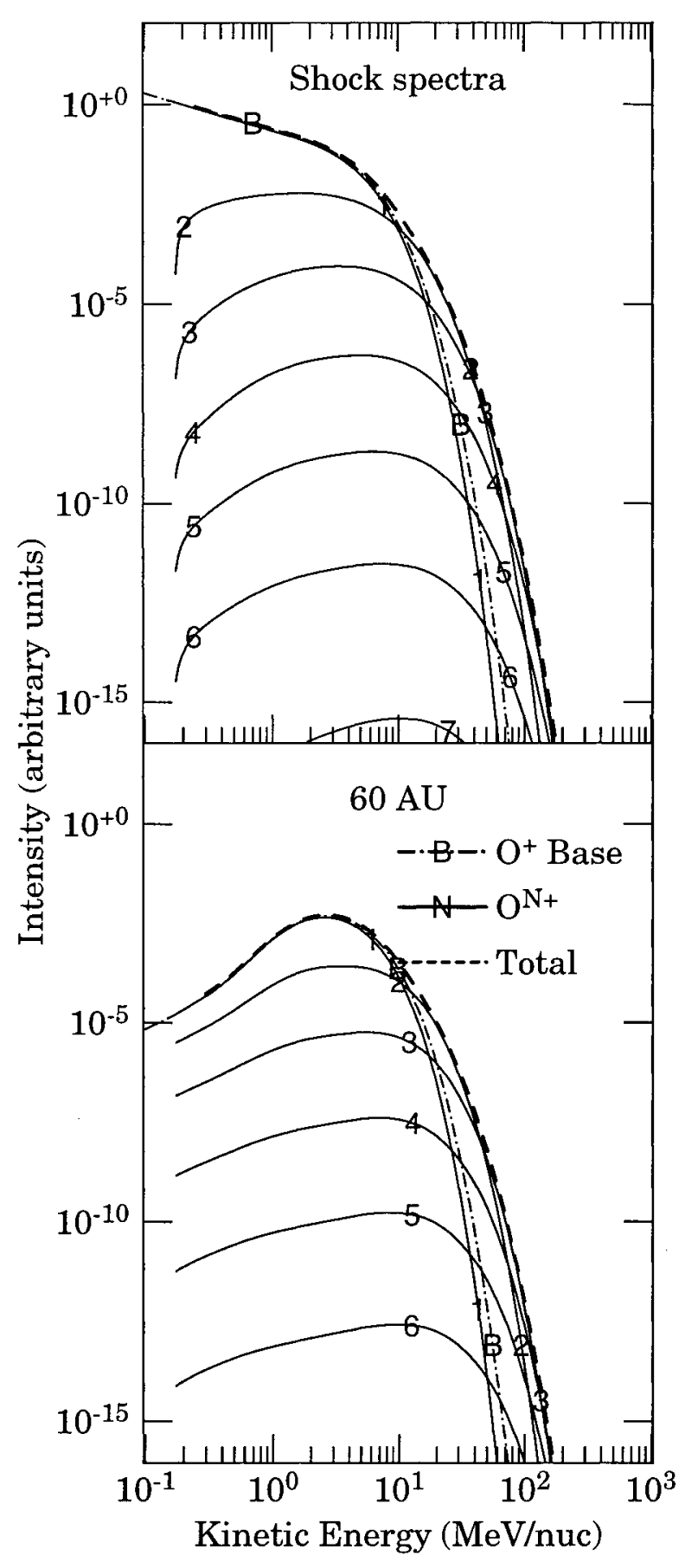

FIGURE 4. ACR $\mathrm{O}^{+} \ldots \mathrm{O}^{8+}$ shock spectra (top) and at $60 \mathrm{AU}$ (bottom). The number on each curve is the charge state, the dashed line is the total of all 8 charge states. The line marked $B$ is the baseline solution. 


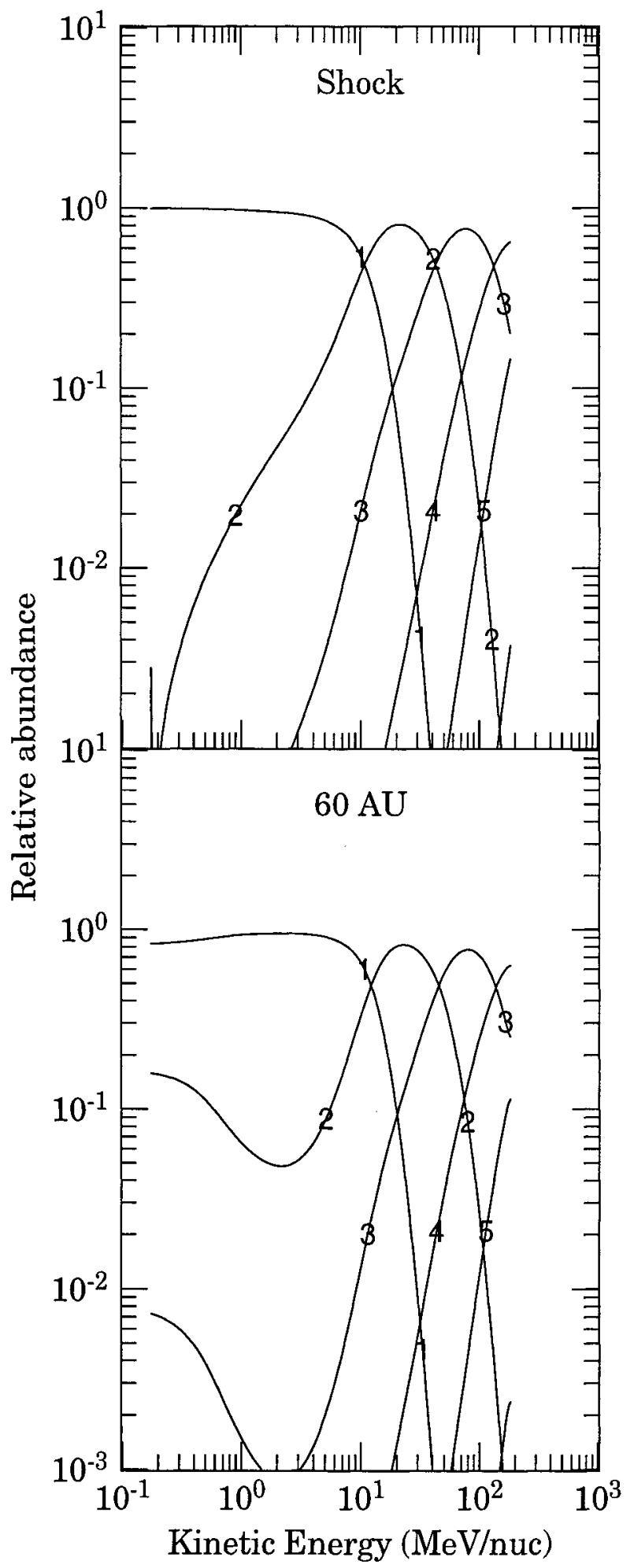

FIGURE 5. $\mathrm{ACR} \mathrm{O}^{+} \ldots \mathrm{O}^{8+}$ relative abundances at the shock (top) and at $60 \mathrm{AU}$ (bottom). erated more efficiently at the SWTS, reaching higher energies (in energy per nucleon) than singly charged particles. In Figure 5 we show that at the shock, $\mathrm{O}^{2+}$ becomes more abundant than $\mathrm{O}^{+}$at $10 \mathrm{MeV} /$ nuc $(11 \mathrm{MeV} / \mathrm{nuc}$ at $60 \mathrm{AU})$, while $\mathrm{O}^{3+}$ in turn becomes more abundant at 40 $\mathrm{MeV} /$ nuc (44 MeV/nuc at $60 \mathrm{AU}$ ). These 'crossovers' are a natural consequence of charge dependence of the acceleration cutoff.

Although ionization is a relatively unimportant modulation mechanism compared to convection, diffusion, adiabatic energy losses and acceleration, it is a significant source of particles for the acceleration mechanism at the shock. The sum of the accelerated spectra of all charge state can be a significantly broader spectrum compared to a similar spectrum calculated for singly charged ions without taking ionization into account. This is expected to be particularly important for interpreting observations of ACRs with larger ionization cross-sections. The contribution of multiply charged ACRs should be more easily detectable for species where the GCRs are significantly less abundant than ACRs.

\section{DRIFT EFFECTS}

During the solar minimum period, drift effects are expected to play an important role in both GCR and ACR modulation, [5] and references therein. The effects of drifts at current sheets on accelerated spectra was described in detail by [13], and could be summarized as follows: a) Accelerated spectra are enhanced (depleted) near current sheets where particles drift upwind (downwind), b) The spatial extent of the enhancement/depletion increases with particle energy and c) the effects should be more pronounced for electrons than protons.

In the heliosphere, positive drift cycle, positively charged particles drifts outward along the wavy neutral sheet, towards the poles along the shock surface, and inward from the poles to the sun in the positive drift cycle,. That is, the drift motion of particles are downstream near the ecliptic, and upstream over the pole in this cycle. We therefore expect an enhancement of the accelerated spectrum over the pole and a depletion near the ecliptic.

To demonstrate the drift effects, equation (1) is solved for an axisymmetric two-dimensional heliosphere, divided into 30 equally spaced intervals, assuming isotropic diffusion in the radial and latitudinal $(\theta)$ directions (i.e. assuming $\lambda_{\theta \theta}=\lambda_{r r}$ ), and a modified [12] Parker spiral magnetic field, with magnitude $B_{e}=5 \mathrm{nT}$ at earth. The diffusion parameters and the solar wind were multiplied by $l(\theta)=1.5+0.5 \cos 2 \theta$, thereby increasing their magni- 
tude by a factor of 2 over the poles relative to the ecliptic. A simulated wavy neutral sheet with a tilt angle of $10^{\circ}$ was used to approximate solar minimum conditions.

A comparison of the drift solution with the baseline solution is shown in Figure 6. At the shock, the ecliptic spectrum is depleted by $20 \%$ relative to the no-drift baseline at the cutoff energy of $5.2 \mathrm{MeV} / \mathrm{nuc}$, while the spectrum at the pole is enhanced by a factor of 4 at this energy, taking the latitudinal gradient into account. In both cases, the modification does neither destroy the power law shape of the spectra at low energies, nor is the cutoff energy significantly modified. For our choice of parameters, the modulated ACR spectra at $60 \mathrm{AU}$ are not substantially modified by drift effects.

\section{SUMMARY}

One-and two-dimensional solutions of the cosmic ray transport equation was used to demonstrate important characteristics of ACR spectra accelerated at the SWTS, which can be summarized as follows:

- Modulated ACR spectra have a peak intensity that is determined by the cutoff in the spectrum at the SWTS.

- ACR spectra at the SWTS have a characteristic power law form, with a spectral index determined by the compression ratio of the shock.

- This power law extends to a certain cutoff energy, which occurs at an energy where $V r_{s}=C_{c} \kappa_{r r}$. Defining the cutoff to be where the spectrum has fallen to $1 / e$ of its power law extrapolation, it is found that $C_{c} \sim 4.5$.

- The species dependence of the diffusion and drift terms in the diffusion tensor and the dependence of the cutoff in the shock spectra on $\kappa_{r r}$ give rise to species dependent modulation, or species scaling, of ACRs.

- The power law spectrum may be modified by drift effects, which either enhances of suppresses acceleration, depending on the direction of the particle drift motion relative to the direction of convection by the solar wind.

- ACRs are ionized by collisions with neutral hydrogen atoms in the heliosphere, giving rise to multiply charged ions.

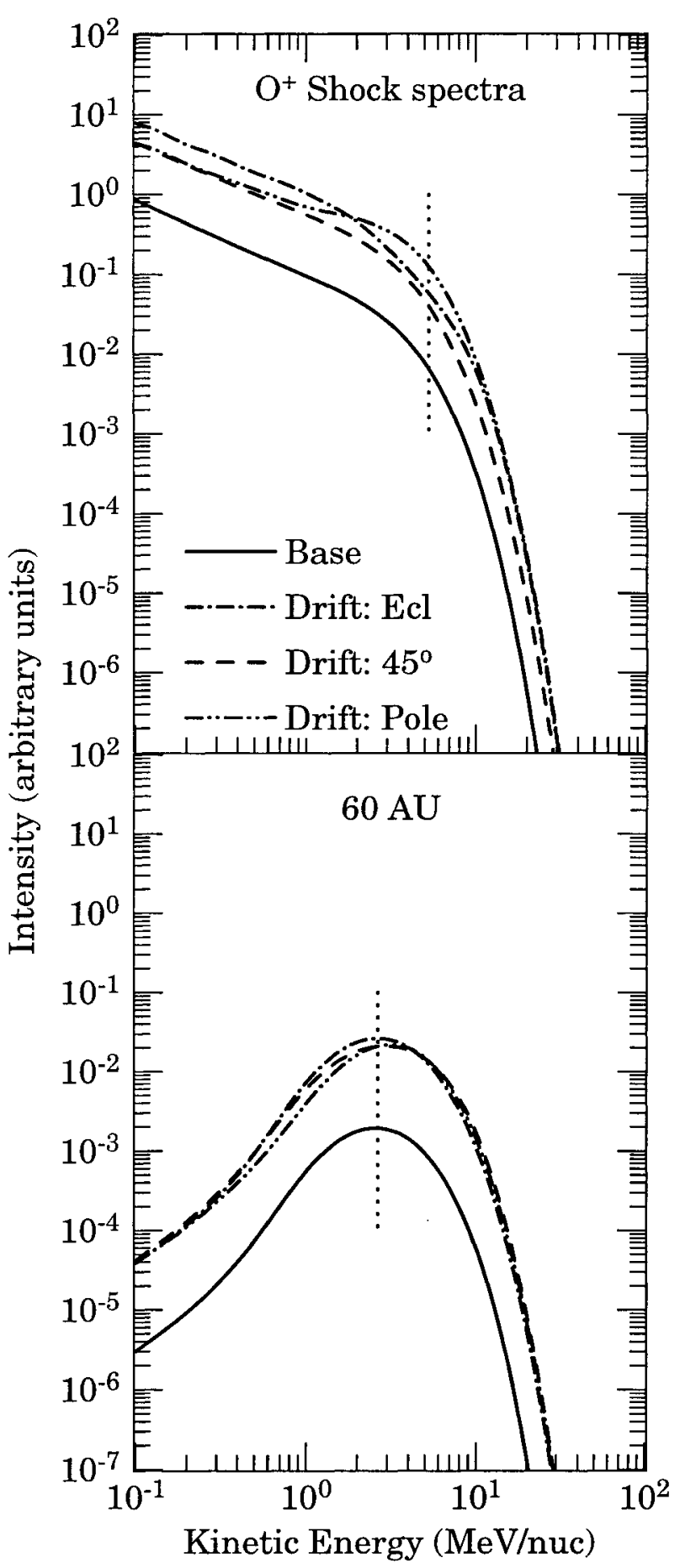

FIGURE 6. $\mathrm{ACR} \mathrm{O}^{+}$shock spectra (top) and at $60 \mathrm{AU}$ (bottom) comparing the no-drift baseline spectra (solid lines) with driftmodified spectra in the ecliptic (dashes), at $45^{\circ}$ latitude (dashdot), and at the pole (dash-dot-dot). Vertical lines (dots) show the cutoff at 5.2 MeV/nuc (top), and the spectral peaks at $\sim 2.6$ $\mathrm{MeV} /$ nuc. The drift spectra are offset by a factor of 10 relative the the baseline spectra. 
- Multiply charged ACRs give rise to modulated spectra that are broader in energy than those consisting only of singly charged ACRs.

\section{ACKNOWLEDGEMENT}

This work was supported by NASA at Caltech (contract NAS7-918).

\section{REFERENCES}

1. Barghouty, A.F., Robust estimates of hydrogen-impact ionization cross sections over a wide energy range, Phys. Rev. $A, 61$, nr. 052702 (2000)

2. Barghouty, A.F., Jokipii, J.R., Mewaldt, R.A., Acceleration, ionization, and transport of multiply charged anomalous cosmic rays: interpretation of SAMPEX observations, This volume (2000)

3. Blandford, R., Eichler, D., Particle acceleration at astrophysical shocks: a theory of cosmic ray origin, Physics Reports, 154(1), 1 (1987)

4. Cummings, A.C., Stone, E.C., Webber, W.R., Evidence that the anomalous cosmic-ray component is singly ionized, Astrophys. J. Lett, 287, L99 (1984)

5. Fisk, L.A., An overview of the transport of galactic and anomalous cosmic rays in the heliosphere: Theory, Adv. Space Res., 23, 415 (1999)

6. Fisk, L.A., Kozlovsky, B., Ramaty, R., An interpretation of the observed oxygen and nitrogen enhancements in lowenergy cosmic rays, Astrophys. J., 190, L35 (1974)

7. Gloeckler, G., Fisk, L.A., Geiss, J., Anomalously small magnetic field in the local interstellar cloud, Nature, 386, 374 (1997)

8. Jokipii, J.R., Particle drift diffusion, and acceleration at shocks, Astrophys. J. Lett., 255, 716 (1982)

9. Jokipii, J.R., Rate of energy gain and maximum energy in diffusive shock acceleration, Astrophys. J. Lett., 313, 842 (1987)

10. Jokipii, J.R., Constraints on the acceleration of anomalous cosmic rays, Astrophys. J. Lett., 393, L41 (1992)

11. Jokipii, J.R., Theory of multiply charged anomalous cosmic rays, Astrophys. J. Lett., 466, L47 - L50 (1996)

12. Jokipii, J.R., Kóta, J., The polar heliospheric magnetic field, Geophys. Res. Lett., 16, 1 (1989)

13. Kota, J., Jokipii, J.R., Diffusive shock acceleration in the presence of current sheets, Astrophys. J., 429, 385 (1994)

14. Klecker, B., Mewaldt, R., et al., A search for minor ions in anomalous cosmic rays, Space Sc. Rev., 83, 259 (1998)
15. Mewaldt, R.A., Selesnick, R.S., Cummings, J.R., Stone, E.C., von Rosenvinge, T.T., Evidence for multiply charged anomalous cosmic rays, Astrophys. J. Lett., 466, L43 (1996)

16. Pesses, M.E., Jokipii, J.R., Eichler, D., Cosmic ray drift, shock-wave acceleration, and the anomalous component of cosmic rays, Astrophys. J. Lett., 246, L85 (1981)

17. Moraal, H., Steenberg, C.D., Basic properties of anomalous cosmic ray spectra, Proc. 26th Int. Cosmic Ray Conf. (Salt Lake), 7, 543 (1999)

18. E.N. Parker, The passage of energetic particles through interplanetary space, Planet. Space Sci, 13, 9 (1965)

19. Steenberg, C.D., Cummings, A.C., Stone, E.C, Drift calculations on the modulation of anomalous cosmic rays during the 1998 solar minimum period, Proc. 26th Int. Cosmic Ray Conf. (Salt Lake), 7, 593 (1999)

20. Steenberg, C.D., Moraal, H., Form of the anomalous cosmic ray spectrum at the solar wind termination shock, $J$. Geophys. Res., 104(A11), 24879 (1999)

21. Steenberg, C.D., Moraal, H., McDonald F.B., Species scaling of modulated anomalous cosmic ray spectra, Proc. $25 \mathrm{th}$ Int. Cosmic Ray Conf. (Durban), 2, 233 (1997)

22. Steenkamp, R., Shock acceleration as source of the anomalous component of cosmic rays in the heliosphere, Ph.D. Thesis, Potchefstroom University for CHE, South Africa (1995)

23. Stone, E.C., Cummings, A.C., Webber, W.R, The distance to the termination shock in 1993 and 1994 from observations of anomalous cosmic rays, J. Geophys. Res., 101(A5), 11017 (1996) 